\title{
Coexistence of magnetic order and charge density wave in a Kondo lattice: $\mathbf{Y b}_{5} \mathbf{I r}_{4} \mathrm{Si}_{10}$
}

\author{
Z. Hossain,* M. Schmidt, W. Schnelle, H. S. Jeevan, C. Geibel, S. Ramakrishnan, ${ }^{\dagger}$ J. A. Mydosh, ${ }^{\ddagger}$ and Y. Grin \\ Max Planck Institute for Chemical Physics of Solids, Nöthnitzer Straße 40, 01187 Dresden, Germany
}

(Received 19 October 2004; published 22 February 2005)

\begin{abstract}
We have examined the ground-state properties of the putative intermediate valency and/or charge density wave (CDW) system $\mathrm{Yb}_{5} \mathrm{Ir}_{4} \mathrm{Si}_{10}$ via resistivity, specific heat, magnetic susceptibility, and $\mathrm{x}$-ray absorption spectroscopy. The bulk properties confirm a CDW-type anomaly at $48 \mathrm{~K}$, but show that all the $\mathrm{Yb}$ ions are in an integral trivalent $4 f^{13}$ state. Additionally, our low-temperature measurements reveal two magnetic transitions at 2.3 and $1.1 \mathrm{~K}$, respectively. The compound shows Kondo-lattice-type behavior that might be responsible for the weak-coupling nature of the CDW evidenced by the specific heat data.
\end{abstract}

DOI: 10.1103/PhysRevB.71.060406

PACS number(s): 75.20.Hr, 75.50.Ee, 71.45.Lr

The study of multiple coexisting or competing types of phases is a frontier area of condensed matter physics. $\mathrm{Nu}-$ merous investigations have been made to understand the interplay of magnetism, superconductivity, giant magnetoresistance, and charge density wave (CDW) ordering in inorganic and organic compounds. Many of these materials exhibit low-dimensional [one-(1D) or two-dimensional (2D)] structure elements and this property is thought to be central to the appearance of these unusual ground states. Thus, threedimensional (3D) materials that display a CDW are especially interesting. The compounds of this kind, then, should have a highly anisotropic Fermi surface that supports a CDW.

Recent studies have shown that a class of intermetallic compounds $R_{5} \mathrm{Ir}_{4} \mathrm{Si}_{10}(R=\mathrm{Dy}-\mathrm{Lu}$ and $\mathrm{Y})$ exhibit the coexistence of the CDW with magnetism or superconductivity. ${ }^{1-6}$ In particular, a high-quality single crystal of $\mathrm{Lu}_{5} \mathrm{Ir}_{4} \mathrm{Si}_{10}$ shows the formation of a commensurate CDW along the $c$ axis below $80 \mathrm{~K}$ in the $(h 0 l)$ plane that coexists with BCS-type superconductivity below $3.9 \mathrm{~K} .{ }^{2}$ Further on, in a single crystal of $\mathrm{Er}_{5} \mathrm{Ir}_{4} \mathrm{Si}_{10}$, one observes the development of a $1 \mathrm{D}$ incommensurate $\mathrm{CDW}$ at $155 \mathrm{~K}$, which then locks into a purely commensurate state below $55 \mathrm{~K}^{3}$ Eventually the localized $\mathrm{Er}^{3+}$ moments order antiferromagnetically (AFM) below $2.8 \mathrm{~K}$, which results in the coexistence of strongly coupled CDW with local moment AFM. Unlike conventional CDW systems, very sharp changes in all bulk properties along with huge heat capacity peaks make this CDW transition a most interesting one and suggest a strong lattice coupling. Studies on homologues with other heavy rare-earth elements also indicate the presence of CDW transitions, which seem to occur at higher temperatures as the cell volume increases. ${ }^{1,4,5}$ Yet, in $\mathrm{Lu}_{5} \mathrm{Ir}_{4} \mathrm{Si}_{10}$, when $\mathrm{Ir}$ is replaced with $\mathrm{Rh}$ or $\mathrm{Si}$ with $\mathrm{Ge}$, the CDW disappears. ${ }^{7,8}$

A notable exception to this generic behavior is the controversial situation in $\mathrm{Yb}_{5} \mathrm{Ir}_{4} \mathrm{Si}_{10}$. Early resistivity investigations on a polycrystalline sample revealed a small anomaly around $50 \mathrm{~K}$ that was attributed to a CDW or spin-density wave transition. However, the putative CDW transition temperature $\left(T_{\mathrm{CDW}}\right)$ was lower than in both Tm and Lu samples and the valence fluctuation of $\mathrm{Yb}$ ions was claimed to be responsible for that. ${ }^{1,4}$ Furthermore, no magnetic ordering could be detected down to $52 \mathrm{mK}$. The resistivity anomaly at $\approx 50 \mathrm{~K}$ in the $\mathrm{Yb}_{5} \mathrm{Ir}_{4} \mathrm{Si}_{10}$ samples was rather weak and unconfirmed by bulk properties. These samples were prepared by arc melting. It is very difficult to prepare single-phase material using this technique since $\mathrm{Yb}$ has a high vapor pressure and an uncontrollable loss of $\mathrm{Yb}$ occurs, usually causing additional phases to be formed. In order to determine the valency of the $\mathrm{Yb}$, the subtle nature of the phase transition at $\approx 50 \mathrm{~K}$ and possible correlation effects (Kondo, magnetic transitions) at lower temperatures, one needs samples of higher quality. We decided to prepare such and reinvestigate the basic properties after first establishing the $\mathrm{Yb}$ valency. $\mathrm{Yb}_{5} \mathrm{Ir}_{4} \mathrm{Si}_{10}$ provides a unique example in which a CDW transition is found to coexist with the Kondo effect and eventual AFM ordering of $\mathrm{Yb}$ ions.

The polycrystalline samples were prepared using a solidstate reaction. The sensitivity of ytterbium to air and moisture required that the sample preparation was carried out in an argon-filled glove box $\left[p\left(\mathrm{O}_{2}, \mathrm{H}_{2} \mathrm{O}\right) / p(\mathrm{Ar})<10^{-6}\right]$. The three elements in stoichiometric ratio (total mass $1.5 \mathrm{~g}$ ) were sealed in a tantalum crucible using arc welding. The Ta container was heated to $1250{ }^{\circ} \mathrm{C}$ at a rate of $300{ }^{\circ} \mathrm{C} / \mathrm{h}$ and held at this temperature for four days under dynamic vacuum. Afterwards it was cooled to room temperature at $300{ }^{\circ} \mathrm{C} / \mathrm{h}$. In a second step, the sample was ground into powder, pressed into a small pellet, and after sealing it inside a tantalum container, the heat treatment was repeated for four days at $1250{ }^{\circ} \mathrm{C}$.

The samples were characterized by $\mathrm{x}$-ray powder diffraction (XRD) using an image plate camera [HUBER G670, $\mathrm{CuK}_{\alpha 1}$ radiation $(\lambda=1.540598 \AA)$, standard $\mathrm{LaB}_{6} \quad(a$ $\left.=4.1569 \AA), 10^{\circ}<2 \Theta<90^{\circ}, 6 \times 15 \mathrm{~min}\right)$. The sample was loaded between two polyimide foils in an aluminum cell to exclude moisture and oxygen. The lattice parameters as refined on the basis of XRD data are: structure type $\mathrm{Sc}_{5} \mathrm{Co}_{4} \mathrm{Si}_{10},{ }^{9}$ space group $P 4 / m b m, a=12.485(1) \AA$ A, $c$ =4.1806(5) $\AA$. Our lattice parameters match nicely with $a$ $=12.503 \AA, c=4.182 \AA$ (Ref. 1). The values for $\mathrm{Yb}_{5} \mathrm{Ir}_{4} \mathrm{Si}_{10}$ are in between those of the $\mathrm{Lu}$ and of the Tm homologue, giving already doubt to the claim of a mixed valent $\mathrm{Yb}$ state. The best $\mathrm{Yb}_{5} \mathrm{Ir}_{4} \mathrm{Si}_{10}$ sample used for all measurements contained less than $5 \%$ of impurity phases ( $\mathrm{IrSi}$ and $\mathrm{IrSi}_{3}$ ).

The magnetic susceptibility was measured between 2 and $300 \mathrm{~K}$ in various magnetic fields up to $7 \mathrm{~T}$ in a Quantum 


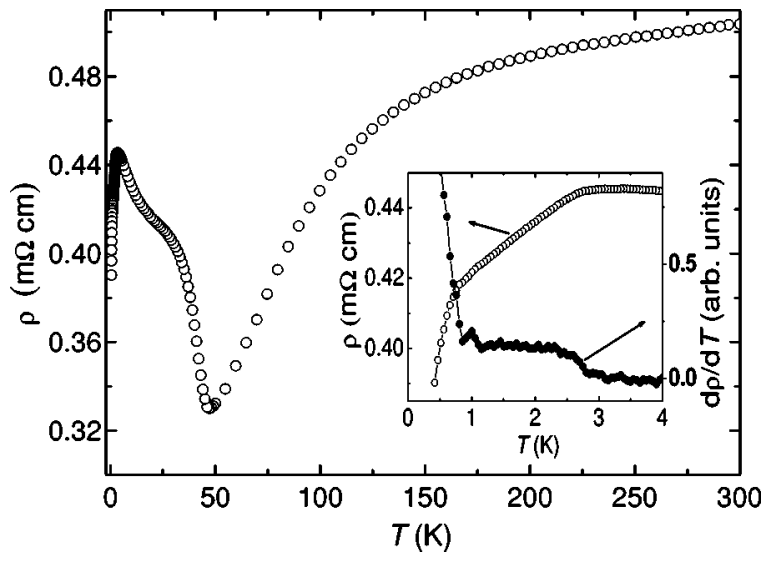

FIG. 1. Overall temperature dependence of the resistivity $\rho(T)$ of $\mathrm{Yb}_{5} \mathrm{Ir}_{4} \mathrm{Si}_{10}$. Inset: The expanded view down to $T=0.4 \mathrm{~K}$ for $\rho$ and $d \rho / d T$ illustrating the magnetic transitions.

Design SQUID magnetometer. Resistivity, magnetoresistance, and heat capacity measurements in the temperature range $0.4<T<300 \mathrm{~K}$ were carried out in a physical property measurement system (Quantum Design) using a fourcontact ac and a relaxation technique, respectively. $\mathrm{Yb} L_{\mathrm{III}}$ $\mathrm{X}$-ray appearance near-edge structure (XANES) spectra were taken at $300 \mathrm{~K}$ in transmission geometry at the EXAFS-II beam line E2 of HASYLAB at DESY, Hamburg, Germany. The wavelength selection was realized by a $\mathrm{Si}(111)$ doublecrystal monochromator, which allowed an experimental resolution of approximately $2 \mathrm{eV}$ at the $\mathrm{Yb} L_{\mathrm{III}}$ edge of $8944 \mathrm{eV}$.

In Fig. 1, we present the temperature dependence of the resistivity $\rho(T)$ of $\mathrm{Yb}_{5} \mathrm{Ir}_{4} \mathrm{Si}_{10}$. The large absolute value of $\rho$ is caused by the graininess and porosity of the sample, which cannot be avoided with our preparation route. In the range $50-300 \mathrm{~K}$, the general behavior corresponds to those of Kondo lattice compounds. ${ }^{10} \mathrm{~A}$ weak temperature dependence ${ }^{11}$ between $150-300 \mathrm{~K}$ is followed by a rapid decrease of $\rho(T)$ below $150 \mathrm{~K}$, related to the vanishing of Kondo scattering by the excited crystal field levels. $\rho(T)$ shows a sudden increase at $50 \mathrm{~K}$, followed by a plateau and then another increase with decreasing temperature between 15 and $3 \mathrm{~K} . \rho(T)$ again decreases below $3 \mathrm{~K}$. Two welldefined anomalies are observed at $\approx 2.5$ and $1.0 \mathrm{~K}$ (see inset of Fig. 1). The sharp increase at $50 \mathrm{~K}$ is very similar to the anomaly observed at the onset of the CDW in the other $R_{5} \mathrm{Ir}_{4} \mathrm{Si}_{10}$ compounds and can therefore be attributed to the CDW transition leading to the opening of a partial gap. A kink in the temperature dependence of the thermoelectric power at $48 \mathrm{~K}$ (see Ref. 4) may be interpreted the same way.

The increase of $\rho(T)$ with decreasing temperature between 15 and $5 \mathrm{~K}$ is typically observed in Kondo lattices showing the magnetic order at lower temperatures and thus gives further evidence for a sizable Kondo effect in this compound. The eventual decrease of resistivity and the concomitant anomalies at $\approx 2.5$ and $1.0 \mathrm{~K}$ are due to the disappearance of spin-disorder scattering associated with the magnetic transitions as established by the specific heat results (see below). Direct evidence for a magnetic-ordered state at low temperatures stems from a large, nonmonotonous magnetoresistance (not shown), which first increases slightly with increasing

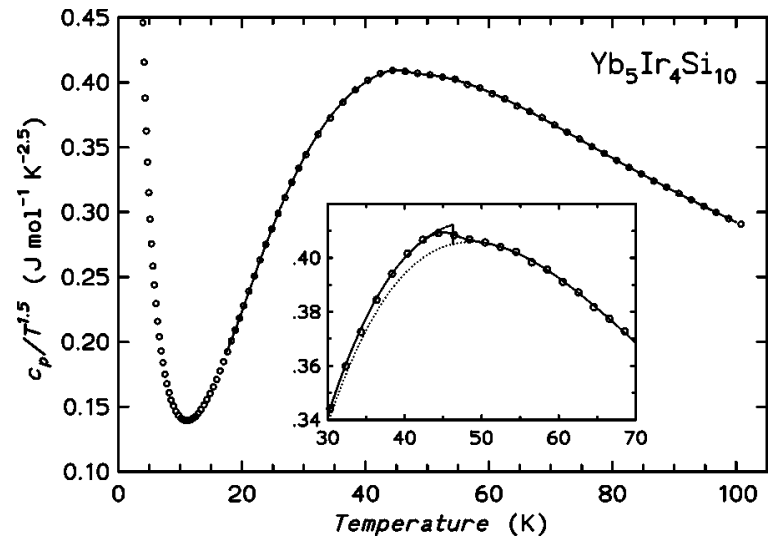

FIG. 2. Specific heat capacity of $\mathrm{Yb}_{5} \mathrm{Ir}_{4} \mathrm{Si}_{10}$ around the CDW transition in a $c_{p} / T^{1.5}$ representation. The inset shows a magnification of the main panel. The full line represents the BCS fit, and the dotted line its phonon and electronic background contribution. The jump at $T_{\mathrm{CDW}}$ (dashed vertical line) would be the idealized sharp CDW transition.

field, before showing at $0.15 \mathrm{~T}$ a pronounced drop indicating a metamagnetic transition, similar to that observed in $\mathrm{Er}_{5} \mathrm{Ir}_{4} \mathrm{Si}_{10} .{ }^{12}$

The specific heat $c_{p}(T)$ around the CDW transition is plotted in Fig. 2 in a special representation that enables us to make visible the small and broadened transition anomaly. In order to quantify the size of the anomaly, a technique, which proved useful in the analysis of the small heat capacity anomalies of high $T_{\mathrm{c}}$ and other superconductors, is utilized. ${ }^{13}$ $c_{p}(T)$ from 17.5 to $100 \mathrm{~K}$ is modeled by the sum of a phonon contribution (a smooth fifth-degree polynomial) plus an electronic term related to the $\mathrm{CDW}$, which we simulated by a weak-coupling BCS temperature dependence. ${ }^{14}$ The fit parameters for the latter contribution are an electronic specific heat coefficient $\gamma$ and $T_{\mathrm{CDW}}$. The step in $c_{p}(T)$ at $T_{\mathrm{CDW}}$ was convoluted with a Gaussian to mimic a transition broadened by inhomogeneities (parameter $\Delta T / T_{\mathrm{CDW}}$ ). An excellent fit was obtained with $\gamma=34(3) \mathrm{mJ} \mathrm{mol}^{-1} \mathrm{~K}^{-2}$, $T_{\mathrm{CDW}}=46.3(5) \mathrm{K}$, and $\Delta T / T_{\mathrm{CDW}}=0.021(1)$. The fitted curve, as well as the idealized "jump" $\Delta c_{p}$ of size $1.43 \gamma T_{\mathrm{CDW}}$ $=2.3 \mathrm{~J} \mathrm{~mol}^{-1} \mathrm{~K}^{-1}$, are plotted in the inset of Fig. 2. The small $\Delta c_{p}$ is a measure of the Fermi surface modification at the CDW transition and not related to the electronic-magnetic specific heat observed at low temperatures. The weakcoupling-type anomaly we observe in $\mathrm{Yb}_{5} \mathrm{Ir}_{4} \mathrm{Si}_{10}$ contrasts with the huge $\lambda$-type or even first-order-type anomaly observed in $\mathrm{Er}_{5} \mathrm{Ir}_{4} \mathrm{Si}_{10}$ and in $\mathrm{Lu}_{5} \mathrm{Ir}_{4} \mathrm{Si}_{10}$, respectively., ${ }^{2,3}$ One could attribute this difference to disorder in the polycrystalline $\mathrm{Yb}_{5} \mathrm{Ir}_{4} \mathrm{Si}_{10}$ sample, but the relative change $[\Delta \rho$ $-\rho(T)] / \rho(T)$ related with the CDW is not significantly smaller in this sample than in the $\mathrm{Er}_{5} \mathrm{Ir}_{4} \mathrm{Si}_{10}$ and $\mathrm{Lu}_{5} \mathrm{Ir}_{4} \mathrm{Si}_{10}$ single crystals. We therefore suspect this weak-coupling-type CDW is intrinsic and related to a much weaker coupling of the CDW to the lattice due to the hybridization between conduction and $4 f$ electrons connected with the Kondo effect. This hybridization would also reduce the heat capacity anomaly by suppression of the CDW energy gap.

The low-temperature specific heat shows two anomalies 


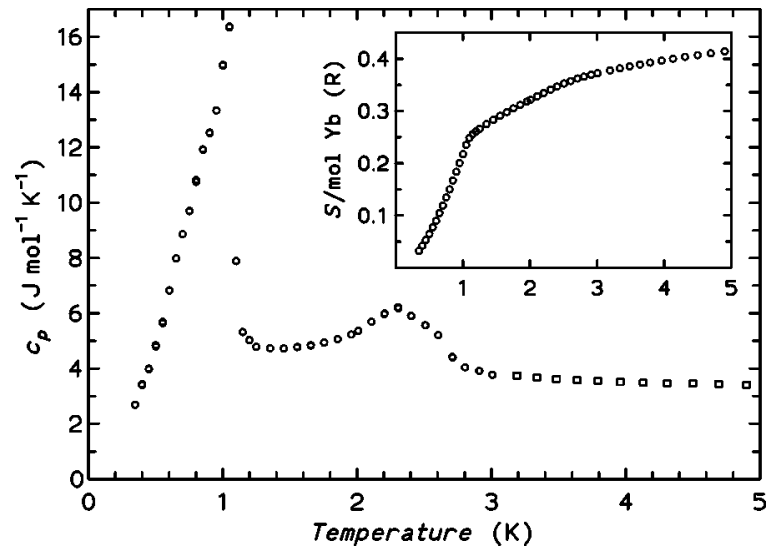

FIG. 3. Specific heat of $\mathrm{Yb}_{5} \mathrm{Ir}_{4} \mathrm{Si}_{10}$ at low temperature showing the two magnetic transitions. Inset: Entropy per mol $\mathrm{Yb}$ (gas constant $\left.R=k_{\mathrm{B}} N_{\mathrm{A}}\right)$.

due to the magnetic transitions, a rather small and broadened one at $\approx 2.3 \mathrm{~K}\left(T_{\mathrm{N} 1}\right)$ and a much larger and sharper $\lambda$-like peak at $1.1 \mathrm{~K}\left(T_{\mathrm{N} 2}\right)$, see Fig. 3. Since the $\mathrm{Yb}$ ions occupy three nonequivalent lattice sites and thus could posses different magnetic-moment interactions and ordering temperatures, the magnetic transitions are expected to be multiple and the magnetic structures complex. All the other isostructural $R_{5} \mathrm{Ir}_{4} \mathrm{Si}_{10}$ compounds with $R=\mathrm{Dy}, \mathrm{Ho}, \mathrm{Er}$, and $\mathrm{Tm}$, which exhibit CDW transitions at high temperatures, also exhibit two successive magnetic transitions at low temperature. ${ }^{15,16}$ The magnetic ordering temperatures of the $\mathrm{Yb}$ compound are close to those of $\mathrm{Er}_{5} \mathrm{Ir}_{4} \mathrm{Si}_{10}$.

As seen from Fig. 3, $\gamma=c_{p} / T$ attains a large value of $0.25 \mathrm{~J} \mathrm{~mol}^{-1} \mathrm{~K}^{-2}$ at $3 \mathrm{~K}$ just before the magnetic ordering begins. Such a huge $\gamma$ indicates the formation of a strongly correlated electron (heavy fermion) state that occurs usually through the Kondo effect.

The peak height of the anomaly in $c_{p}(T)$ at $T_{\mathrm{N} 2}$ is only $3.5 \mathrm{~J}(\mathrm{~mol} \mathrm{Yb})^{-1} \mathrm{~K}^{-1}$, which is much smaller than $\approx 12 \mathrm{~J}(\mathrm{~mol} \mathrm{Yb})^{-1} \mathrm{~K}^{-1}$ expected for a mean field transition in a well-localized $S=1 / 2$ system. In many Ce-based Kondo systems, a reduced value of the jump height and magnetic entropy are observed. ${ }^{17}$ We believe a similar situation exists here as well. The magnetic entropy amounts to $S_{1}=0.35 R \ln 2 /(\mathrm{mol} \mathrm{Yb})$ at $T_{\mathrm{N} 1}$ and to $S_{2}$ $=0.5 R \ln 2 /(\mathrm{mol} \mathrm{Yb})$ at $T_{\mathrm{N} 2}$. This indicates that only the ground state crystal field doublet contributes at these temperatures, but due to the Kondo effect the magnetic entropy at $T_{\mathrm{N}}$ is at least $50 \%$ lower than $R \ln 2$. Based upon resistivity, susceptibility (see below) and specific heat data we estimate a Kondo temperature $T_{\mathrm{K}}$ of order of $15 \mathrm{~K}$. The much larger absolute value of the thermopower of the $\mathrm{Yb}$ compound as compared to its homologues, as well as its negative sign, ${ }^{4}$ are further support for a signficant Kondo interaction.

The anomalies at $T_{\mathrm{N} 1}$ are rather small and can therefore not be excluded to originate from a foreign phase. The detected binary compounds $\mathrm{IrSi}$ and $\mathrm{IrSi}_{3}$ are, however, nonmagnetic. The size of the specific heat at $T_{\mathrm{N} 2}$ and the entropy associated with this anomaly are so large that one can exclude their origin from a foreign phase, which would have to amount to more than $30 \mathrm{~mol} \%$. Thus, these specific heat

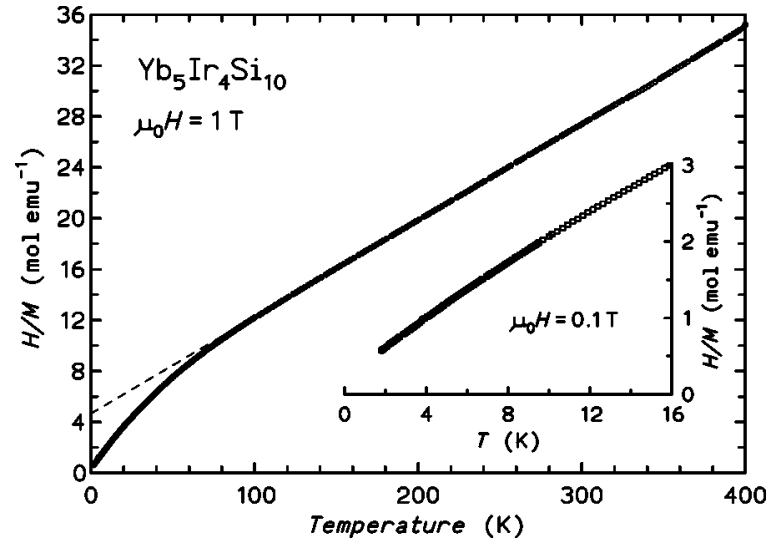

FIG. 4. Inverse magnetic susceptibility $H / M(T)$ of the $\mathrm{Yb}_{5} \mathrm{Ir}_{4} \mathrm{Si}_{10}$ sample measured at $\mu_{0} H=1 \mathrm{~T}$ (inset: $\mu_{0} H=0.1 \mathrm{~T}$ ) from 1.8 to $400 \mathrm{~K}$. The line represents a Curie-Weiss fit $[\chi(T)=C /(T$ $-\Theta)$ ] to the data with $T>100 \mathrm{~K}$.

results give an unambiguous proof of the magnetic order of $\mathrm{Yb}$ in this compound.

The valence state of $\mathrm{Yb}$ was determined from the temperature dependence of the magnetic susceptibility and from x-ray absorption spectroscopy (XAS) results. The earlier study ${ }^{1}$ proposed valence fluctuation to be responsible for the low value of $T_{\mathrm{CDW}}$ in this compound. The inverse magnetic susceptibility $H / M(T)$ is given in Fig. 4 . $\mathrm{Yb}_{5} \mathrm{Ir}_{4} \mathrm{Si}_{10}$ displays the typical behavior of a compound with ytterbium ions in the ${ }^{2} F_{7 / 2}$ crystal field ground multiplet of the $4 f^{13}$ configuration, i.e., stable trivalent $\mathrm{Yb}$. The effective magnetic-moment $\mu_{\mathrm{eff}} / \mathrm{Yb}$ atom $=4.59 \mu_{\mathrm{B}}\left(\mathrm{Yb}^{3+}\right.$ free-ion moment $\left.4.54 \mu_{\mathrm{B}}\right)$ and the Weiss parameter $\Theta=-61.3(1) \mathrm{K}$ (i.e., AFM) obtained from a Curie-Weiss fit above $100 \mathrm{~K}$ are fully compatible with this interpretation (see line in Fig. 4) and imply that all the three $\mathrm{Yb}$ sites in $\mathrm{Yb}_{5} \mathrm{Ir}_{4} \mathrm{Si}_{10}$ are in a trivalent configura-

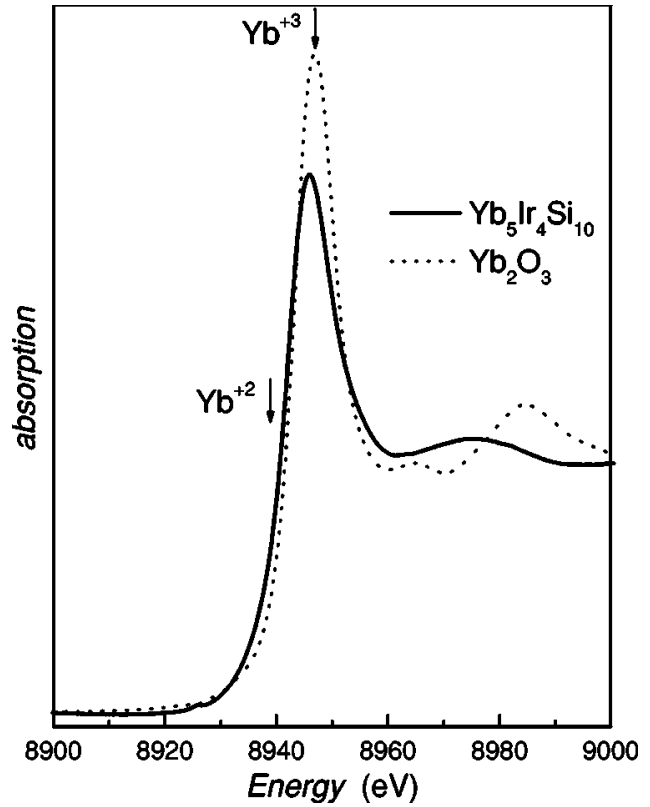

FIG. 5. X-ray absorption spectra ( $\mathrm{Yb} L_{\mathrm{III}}$ edge) of $\mathrm{Yb}_{5} \mathrm{Ir}_{4} \mathrm{Si}_{10}$ and of the reference $\mathrm{Yb}_{2} \mathrm{O}_{3}$ at room temperature. 
tion. The large value of $|\Theta|$ suggests that hybridization between $4 f$ and conduction electrons is significant and consistent with the fact that Kondo interaction $\left(T_{\mathrm{K}} \approx|\Theta / 4|\right.$ $\approx 15 \mathrm{~K})$ dominates the resistivity and specific heat. The deviation from the Curie-Weiss law below $100 \mathrm{~K}$ is typical for crystal field effects in a localized $4 f$ system and related to the smaller moment of the crystal electric field ground state. The absence of any visible anomaly around $46 \mathrm{~K}$ indicates that the $\mathrm{CDW}$ is not connected with any changes in the $\mathrm{Yb}$ valence.

Our XAS results at room temperature confirm that $\mathrm{Yb}$ is in the trivalent state rather than in the valence fluctuating state as previously proposed. ${ }^{1,4}$ The experimental data shown in Fig. 5 were measured simultaneously with $\mathrm{Yb}_{2} \mathrm{O}_{3}$ as an external reference. The $\mathrm{x}$-ray absorption spectrum of $\mathrm{Yb}_{5} \mathrm{Ir}_{4} \mathrm{Si}_{10}$ is dominated by a single-edge structure with a maximum shift of $1 \mathrm{eV}$ compared to the spectrum of the reference. The electronic configuration of $\mathrm{Yb}$ in $\mathrm{Yb}_{5} \mathrm{Ir}_{4} \mathrm{Si}_{10}$ is thus $4 f^{13}\left(\mathrm{Yb}^{3+}\right)$. The absence of a second peak in the spectra illustrates that the sample does not contain measurable amounts of $\mathrm{Yb} 4 f^{14}\left(\mathrm{Yb}^{2+}\right)$. The shift of $1 \mathrm{eV}$ of the maxima relative to the spectrum of $\mathrm{Yb}_{2} \mathrm{O}_{3}$ is caused by the different character of the chemical bonding of $\mathrm{Yb}$ in the oxide compared to $\mathrm{Yb}_{5} \mathrm{Ir}_{4} \mathrm{Si}_{10}$.

In conclusion, through combined bulk measurements on a well-characterized sample, we have resolved the exact nature of the ground state of $\mathrm{Yb}_{5} \mathrm{Ir}_{4} \mathrm{Si}_{10}$. We provide experimental evidence of a charge-density wave transition in a Kondo system, which also undergoes low-temperature magnetic ordering. Our investigations were done on polycrystalline samples. In the Guinier diffraction experiments, we were unable to establish the lattice modulation ( $q$ vectors) arising from the CDW transition. Such were done in both $\mathrm{Lu}_{5} \mathrm{Ir}_{4} \mathrm{Si}_{10}$ and $\mathrm{Er}_{5} \mathrm{Ir}_{4} \mathrm{Si}_{10}$ single crystals using high-intensity $\mathrm{x}$-ray synchrotron radiation and thereby presented proof of CDW. ${ }^{2,3,5}$ Single-crystal growth for such investigations on $\mathrm{Yb}_{5} \mathrm{Ir}_{4} \mathrm{Si}_{10}$ is currently underway and synchrotron diffraction will be pursued in the near future.

We thank K. Klementiev (HASYLAB, Hamburg) for assistance with the XAS measurements and H. Rosner for helpful discussions.
*Permanent address: Department of Physics, Indian Institute of Technology, Kanpur 208016, India.

'Permanent address: Tata Institute of Fundamental Research, Bombay 400005, India.

Permanent address: Kamerlingh Onnes Laboratory, Leiden University, Leiden, The Netherlands.

${ }^{1}$ For earlier references on polycrystalline work, see H. D. Yang, P. Klavins, and R. N. Shelton, Phys. Rev. B 43, 7688 (1991); K. Ghosh, S. Ramakrishnan, and Girish Chandra, Phys. Rev. B 48, 4152 (1993), and references cited therein.

${ }^{2}$ B. Becker, N. G. Patil, S. Ramakrishnan, A. A. Menovsky, G. J. Nieuwenhuys, J. A. Mydosh, M. Kohgi, and K. Iwasa, Phys. Rev. B 59, 7266 (1999).

${ }^{3}$ F. Galli, S. Ramakrishnan, T. Taniguchi, G. J. Nieuwenhuys, J. A. Mydosh, S. Geupel, J. Lüdecke, and S. van Smaalen, Phys. Rev. Lett. 85, 158 (2000).

${ }^{4}$ Y.-K. Kuo, F. H. Hsu, H. H. Li, H. F. Huang, C. W. Huang, C. S. Lue, and H. D. Yang, Phys. Rev. B 67, 195101 (2003).

${ }^{5}$ S. van Smaalen, M. Shaz, L. Palatinus, P. Daniels, F. Galli, G. J. Nieuwenhuys, and J. A. Mydosh, Phys. Rev. B 69, 014103 (2004).

${ }^{6}$ Y. K. Kuo, Y. Y. Chen, L. M. Wang, and H. D. Yang, Phys. Rev. B 69, 235114 (2004).
${ }^{7}$ H. D. Yang, R. N. Shelton, and H. F. Braun, Phys. Rev. B 33, 5062 (1986).

${ }^{8}$ Yogesh Singh and S. Ramakrishnan, Physica B (to be published).

${ }^{9}$ H. F. Braun and K. Yvon, Acta Crystallogr., Sect. B: Struct. Crystallogr. Cryst. Chem. 36, 2397 (1980).

${ }^{10} \mathrm{~J}$. M. Mignot and J. Wittig, in Valence Instabilities, edited by P. Wachter and H. Boppart (North-Holland, Amsterdam, 1982), p. 203.

${ }^{11}$ If we subtract the phonon contribution of the Lu compound above $150 \mathrm{~K}$, we obtain a negative $T$ coefficient of the resistivity for $\mathrm{Yb}_{5} \mathrm{Ir}_{4} \mathrm{Si}_{10}$, i.e., Kondo-like.

${ }^{12}$ F. Galli, Ph.D. thesis, Leiden University, 2002.

${ }^{13}$ R. W. Henn, W. Schnelle, R. K. Kremer, and A. Simon, Phys. Rev. Lett. 77, 374 (1996).

${ }^{14}$ B. Mühlschlegel, Z. Phys. 155, 313 (1959).

${ }^{15}$ F. Galli, R. Feyerherm, R. W. A. Hendrikx, S. Ramakrishnan, G. J. Nieuwnhuys, J. A. Mydosh, Phys. Rev. B 62, 13840 (2000).

${ }^{16}$ F. Galli, R. Feyerherm, R. W. A. Hendrikx, E. Dudzik, S. Ramakrishnan, S. D. Brown, S. van Smaalen, J. A. Mydosh, J. Phys.: Condens. Matter 14, 5067 (2002).

${ }^{17}$ N. Grewe and F. Steglich, in Handbook on the Physics and Chemistry of the Rare Earths, edited by K. A. Gschneidner and L. Eyring (Elsevier, Amsterdam, 1991), Vol. 14, p. 343. 